\title{
Sparing Immunosuppression in Heart Transplant Recipients With Severe Sepsis
}

\author{
N.K. Chou, W.J. Ko, N.H. Chi, Y.S. Chen, H.Y. Yu, R.B. Hsu, C.T. Fang, S.C. Chang, F.Y. Lin, \\ S.H. Chu, and S.S. Wang
}

\begin{abstract}
This study described an analysis of severe sepsis among heart transplantation recipients who were treated by sparing all immunosuppressants. Sepsis leading to multiple organ failure (MOF) in heart transplantation has a high mortality. This retrospective study of 190 patients who underwent heart transplantation from 1993 to 2004 included 12 who had severe sepsis with MOF who were treated by sparing all immunosuppressants. Half of them survived after sparing all immunosuppressants with intensive endomyocardial biopsy. Only one case needed pulse therapy for an acute rejection episode. The most common bacterial infectious episodes were caused by methicillin-resistant Staphylococcus aureus $(n=3)$. All sepsis episodes occurred in the first month after heart transplantation except in one case, which occurred 6 years after heart transplantation. There was a $50 \%$ survival rate of heart transplantation recipients who experienced MOF due to severe sepsis and were treated by sparing all immunosuppressants under a program of intensive endomyocardial biopsy.
\end{abstract}

$\mathrm{I}^{\mathrm{N}}$ NFECTION AND REJECTION are the two primary barriers to successful organ transplantation. ${ }^{1}$ After a couple of days, bacterial, fungal, and viral infections may occur. The level of immunosuppression must be adapted to prevent rejection. Optimal treatment of an organ transplant recipient with severe sepsis is demands careful attention to the immunosuppressive regimen and the antimicrobial strategy. Of organ transplant patients with documented infections, $11.7 \%$ develop severe sepsis, which is the most common reason for intensive care unit utilization. ${ }^{2}$ In particular, signs of infectious disease may be subtle, because of the diminished inflammatory response to infection. Inadequate immunosuppression may lead to rejection, whereas excessive immunosuppression increases the risk of infection. Therefore, thorough surveillance for infectious processes is imperative. ${ }^{3}$ Sepsis leading to multiorgan failure (MOF) in heart transplantation (HTx) has a high mortality. None of the previous studies in the field of HTx patients with severe sepsis have been performed with sparing immunosuppression. This study provided a descriptive analysis of severe sepsis in HTx recipients treated with this strategy.

\section{PATIENTS AND METHODS}

One hundred and ninety patients who underwent HTx from 1993 to 2004 experienced infections, which were listed according to the type of organism, the location of the infection, the time of onset of infection after transplantation, and the clinical outcome. All patients received immunosuppressive therapy according to our HTx protocol. ${ }^{4}$ Perioperative prophylactic antimicrobials included $1 \mathrm{~g}$ of cefazolin given intravenously to HTx recipients every 8 hours until removal of the endotracheal and drain tubes. In an attempt to prevent oropharyngeal candidasis, mycostatin suspension $(5 \mathrm{~mL})$ was given orally four times a day for 1 month after HTx. No prophylactic agents were used to prevent Pneumocystis jirovec or Mycobacterium tuberculosis. Infections were diagnosed by routine methods, after positive culture of the infective agent from an appropriate source, such as blood, wound, sputum, or open lung biopsy. Viral infections were not included in this study, because of the lack of the use of sensitive virological tests. Patient data were analyzed for the presence of severe sepsis plus MOF; ventilator dependence; acute renal failure; hepatic dysfunction; hypoperfusion; or hypotension using the consensus conference definition. ${ }^{5}$

From the Division of Cardiovascular Surgery, Department of Surgery (N.K.C., W.J.K., N.H.C., Y.S.C., H.Y.Y., R.B.H., F.Y.L., S.H.C., S.S.W.) and Internal Medicine (C.T.F., S.C.C.), National Taiwan University Hospital, Taipei, Taiwan.

Address reprint requests to Shoei-Shen Wang, MD, PhD, Department of Surgery, National Taiwan University Hospital, No. 7 Chung-Shan South Road, Taipei, Taiwan. E-mail: sswang@ha. mc.ntu.edu.tw

$0041-1345 / 06 / \$-$ see front matter doi:10.1016/j.transproceed.2006.06.009 
Table 1. Patient Clinical Characteritics

\begin{tabular}{rrrrrllllr}
\hline Case & Days After HTx & T-Bil. & CVVH & Ventilator & Infection Agent & Site & Survival & Rejection & Days of Sparing \\
\hline 1 & 7 & 10.6 & + & + & Candida sp & Blood & No & - & 13 \\
2 & 1800 & 29.9 & - & + & MRSA & Blood & No & - & 6 \\
3 & 17 & 28.6 & + & + & Candida sp & Blood & No & - & 7 \\
4 & 34 & 3.7 & - & + & PDRAB & Sputum & No & - & - \\
5 & 7 & 5.7 & + & + & Pseudomonas & Wound & No & - & 20 \\
6 & 7 & 29 & + & + & Pseudomonas & Blood & No & - & 7 \\
7 & 6 & 14 & + & + & MRSA & Sputum & Yes & - & 3 \\
8 & 7 & 1.7 & + & + & Candida sp & Wound & Yes & - & 2 \\
9 & 11 & 28.2 & + & + & MRSA & Sputum & Yes & + \\
10 & 15 & 3.5 & - & + & Norcardia sp & Lung biopsy & Yes & - & 6 \\
11 & 18 & 3.0 & - & + & Norcardia sp & Lung biopsy & Yes & - & 2 \\
12 & 7 & 11.1 & + & + & PDRAB & Sputum & Yes & - & 5 \\
\hline
\end{tabular}

HTx, heart transplantation; Bil, bilirubin (mg/dL); CVVH, continous venovenous hemofiltration; MRSA, methicillin-resistant Staphylococcus aureus; PDRAB, polydrug-resistant Acinobacter baumannii.

\section{RESULTS}

There were 12 cases $(5.12 \%)$ with severe sepsis with MOF treated temporally without any immunosuppression. Half of them survived after sparing all immunosuppressants with intensive endomyocardial biopsy. Only one case needed pulse steroid therapy for an acute rejection episode. The most common infectious episodes were caused by methicillinresistant Staphylococcus aureus $(n=3)$, Candida $\operatorname{sp}(n=3)$, Norcardia sp $(n=2)$, Pseudomonas aeruginosa $(n=2)$, polydrug-resistant Acinobacter baumannii $(n=2)$. All sepsis episodes occurred in the first month after HTx except in one case that was evident at 6 years after heart transplantation. All patients needed a ventilator for respiratory support. Eleven patients were hyperbilirubinemia (total bilirubin $>3 \mathrm{mg} / \mathrm{dL}$ ) with hepatic dysfunction. Nine patients needed continuous venovenous hemofiltration for acute renal failure. Four patients displayed blood stream infections with a poor prognosis and nonsurvival. The duration of sparing of all immunosuppressants was $8.8 \pm$ 7.3 days among the nonsurvival versus $3.8 \pm 2.5$ days among the survival cohort. The clinical characteristics of the patients are shown in Table 1.

\section{DISCUSSION}

More than $90 \%$ of infections occurring in the first month are nosocomial bacterial or candidal infections in the wound, respiratory tract, urinary tract, or vascular-access devices in HTx recipients. ${ }^{1-3}$ Infection and rejection, the two primary barriers to successful HTx, are inextricably linked. The specific immunosuppressive program of sparing all immunosuppressants with administration of antimicrobials is a strategy for optimal treatment of HTx with severe sepsis-induced MOF. The concept of tailored or "individualized" immunosuppression has been introduced by clini- cians not by basic scientists. Besides an antimicrobial or antifungal program, the level of immunosuppression must be adapted to this challenge while monitoring the risk for rejection. ${ }^{6}$ As new immunosuppressive programs are defined, antimicrobial programs will be necessary. This study was limited to showing the safety of sparing all immunosuppressants. Although cytomegalovirus infection is a major cause of mortality and morbidity after $\mathrm{HTx},{ }^{1-3}$ among our patients its incidence was low $(4.7 \%) .^{5} \mathrm{We}$ need improved diagnostic tests to detect infection early and to monitor immune function, as well as new therapies to overcome antimicrobial resistance.

In conclusion, there was a $50 \%$ survival rate of the HTx recipients who developed MOF due to severe sepsis treated by sparing all immunosuppressants while using an intensive routine of endomyocardial biopsy. In severe sepsis with MOF, the single acute rejection episode did not seem severe after sparing all immunosuppressants.

\section{REFERENCES}

1. Fishman JA, Rubin RH: Infection in organ-transplant recipients. N Engl J Med 338:1741, 1998

2. Trzeciak S, Sharer R, Chan T, et al: Infections and severe sepsis in solid-organ transplant patients admitted from a universitybased ED. Am J Emerg Med 22:530, 2004

3. Stembach GL, Varon J, Hunt SA: Emergency department presentation and care of heart and heart/lung transplant recipients. Am J Emerg Med 21:1140, 1992

4. Shapiro NI, Wolfe RE, Moore RB, et al: Mortality in Emergency Department Sepsis (MEDS) score: a prospectively derived and validated clinical prediction rule. Crit Care Med 31:670, 2003

5. Hsu RB, Fang CT, Chan SC, et al: Infectious complications after heart transplantation in Chinese recipients. Am J Transplant 5:2011, 2005

6. Mohacsi P, Schmidli J, Stalder M: Tailored immunosuppression: the way we should follow? Transplant Proc 34:2944, 2002 